\title{
Rechtsgeschichte
}

http://www.rg-rechtsgeschichte.de/rg7

$\operatorname{Rg} 2005$

$154-157$

Zitiervorschlag: Rechtsgeschichte Rg 7 (2005)

http://dx.doi.org/10.12946/rg07/154-157

\section{Loretana de Libero}

\section{Im Guten wenig und im Schlimmen viel}




\section{Im Guten wenig und im Schlimmen viel*}

Der Henker hatte ganze Arbeit geleistet, der Verurteilte hing am Kreuz. Zwar war der eine Nagel nicht ganz durch das rechte Fersenbein in den Pfahl getrieben worden, so wie es eigentlich hätte sein sollen, aber daran war das harte, knorrige Olivenholz schuld. Vielleicht wurden deshalb die Arme des Delinquenten auch nicht an den Querbalken genagelt, sondern nur gebunden. Ölbäume waren nicht die bevorzugte Holzart für Kreuzigungen, aber geeigneteres Material war wohl in diesem Moment knapp. Letztlich erfüllte die Aufhängung ihren Zweck. Wir wissen nicht, wie lange der Mann am Kreuz hing, aber es scheint, als sei der Henker milde gestimmt gewesen. Die Todesqual beendete er durch das crurifragium, d. h. die Schienbeine des Gekreuzigten wurden zerschlagen, wodurch der Tod unmittelbar eintrat. Die Verwandten des Verurteilten waren nicht ohne Einfluss, wurde doch der Leichnam zur Bestattung freigegeben, statt am Stamm zu verfaulen - aber vielleicht brauchten die Schergen auch einfach nur das Holz für die nächste Hinrichtung. Allerdings hatte der Henker große Mühe, den Toten vom Kreuz abzunehmen, da sich besagter Nagel nun kaum mehr aus dem Holzstamm wieder herausziehen ließ und letztlich herausgebrochen werden musste. Der Eisenstift, der die Schmach der Hinrichtung verriet, verblieb jedoch im Fersenbein, auch bei der späteren Wiederbestattung der Gebeine in einem Ossarium. Es war nämlich nicht möglich, ihn aus dem Knochen herauszuziehen, seine Spitze hatte sich im Holz verbogen. I968 wurden die Überreste dieses Gekreuzigten in Givat'ha-Mivtar nordöstlich von Jerusalem zufällig bei Bauarbeiten entdeckt. Eine Inschrift auf der Knochenkiste nennt seinen Namen:
J'hôhānān (Johannes). Der Fund ist bisher einzigartig. Da sich die Überreste aber in einem schlechten Erhaltungszustand befinden, ist die Art und Weise der Kreuzigung in der Forschung ebenso umstritten wie das crurifragium. Zeitlich wird diese Hinrichtung in das erste nachchristliche Jahrhundert gesetzt (t.a.q. 66/70 n. Chr.), vielleicht geschah sie unter dem kreuzigungswütigen Statthalter Judäas Antonius Felix (ca. 5260 n. Chr.). ${ }^{\mathrm{T}}$

$\mathrm{Ob}$ nun genagelt oder gebunden, die Kreuzigung war ein blutiges Geschäft, ging ihr doch auch eine ausgiebige Geißelung voraus. Aber ob der Fund nun die ultimative Form der Kreuzigung in der Antike zu belegen vermag, wie mancher Forscher in seiner Begeisterung meinte, ist nicht sicher. Darf hierbei doch nicht übersehen werden, dass Johannes von seinem Henker zwar in dieser Art aufgehängt wurde, dies aber nicht zwangsläufig heißen muss, dass grundsätzlich in dieser Weise gekreuzigt wurde bzw. werden musste. Auf der Suche nach »historischen Wahrheiten « wird gelegentlich übersehen, dass menschliche Faktoren eine Rolle spielen: Einfluss auf Entscheidungen kann nämlich auch haben, wie gut der Henker morgens gefrühstückt hatte, ob ihm das Wetter aufs Gemüt drückte oder er sich einfach nur ärgerte über die mindere Qualität des Holzes. Der Phantasie der Schergen waren überdies keine Grenzen gesetzt, sadistische Neigungen konnten hemmungslos ausgelebt werden (Jos. bell. 5, I I.449 ff.).

Recht oder Willkür? Diese Frage müssen wir uns nicht nur für die Niederungen des Richtplatzes stellen, sondern auch für die hohe Politik. Guido Kirner hat nun in seiner Berliner Dissertation aus dem Jahr 2002 den Versuch unter-
Guido O. Kirner, Strafgewalt und Provinzialherrschaft. Eine Untersuchung zur Strafgewaltspraxis der römischen Statthalter in Judäa (6-66 n. Chr.), (Schriften zur Rechtsgeschichte, Bd. I09), Berlin: Duncker \& Humblot 2004, 396 S., ISBN 3-428-I I 38I-O; das Zitat stammt von Theodor Mommsen, Strafrecht, I 899, 237:

옹 "Was die Statthalter gethan haben, im Guten wenig und im
Schlimmen viel, lässt sich durchaus bringen unter den Gebrauch oder Missbrauch der magistratischen Fürsorge für die öffentliche Sicherheit."

I N. HAAs, Anthropological Observations on the Skeletal Remains from Givat'ha-Mivtar, in: Israel Exploration Journal (IEJ) 20 (I970) 49-59; H. W. KuHn, Die Kreuzigungsstrafe während der frühen Kaiserzeit, in: Aufstieg und
Niedergang der Römischen Welt 2, 25.I, 7I I ff. Ergänzungen bzw. Berichtigungen J. ZIAS, E. SEKELES, The Crucified Man from Givat ha-Mivtar, in: IEJ 35 (1985) 22-27. 
nommen, den Charakter der Strafgewalt eines römischen Statthalters am Beispiel Judäas zu untersuchen, der »Provinz «, in der das Kreuz überaus beliebtes Hinrichtungsinstrument war. Ziel seiner Arbeit ist es aufzuzeigen, dass die Maßnahmen des Statthalters weder in ein normatives Strafrechtssystem eingebettet gewesen seien, noch er ein rein »souveränes Herrenrecht " (Mommsen, Strafrecht, 238) ausgeübt habe. Für Kirner agierte der Statthalter in einem »Zwischenbereich rein persönlicher Willkürjustiz und Vollstreckung übergeordneter normativer Rechtsregelungen « (I 5 ). Kern- und Angelpunkt seiner sorgfältig recherchierten Untersuchung ist die statthalterliche Kapitalgerichtsbarkeit. Nach der obligatorischen Kritik an der Mommsenschen Rechtssystematik hinterfragt Kirner dessen strikte Trennung von coercitio und cognitio im Bereich militiae, also von magistratischer Zwangsgewalt und Strafgerichtsbarkeit in den Provinzen. Allerdings müssen wir zur Ehrenrettung des gescholtenen Mommsen festhalten, dass dieser sich der Realität römischer Rechtspraxis durchaus bewusst war, welche in seinem juristischen System aber nichts zu suchen hat, was ihm kaum vorgeworfen werden kann. Anschließend untersucht Kirner die in der Forschung umstrittene These Kunkels zum consilium im magistratischen Kapitalstrafverfahren. Kunkel geht davon aus, dass die Beisitzer des (Pro-)Magistraten nicht nur beratend tätig gewesen seien, wie etwa Mommsen meint, sondern deren Votum darüber hinaus bindende Wirkung besessen habe. Mit dieser Überlegung stellt sich Kunkel überdies gegen Mommsens Vorstellung von einer »selbstherrlichen Gerichtsbarkeit «, da über die Schuld eines Angeklagten das consilium zu entscheiden und der Statthalter nur den Richtspruch zu verkünden habe. Seine These sucht Kirner im ersten Hauptteil der Arbeit (72-I 20) anhand der cice- ronischen Verrinen nachzuweisen, auf die ich wegen ihrer Bedeutsamkeit ein wenig näher eingehen möchte:

Der erste von Kirner behandelte Fall ist der Kapitalprozess gegen Sopatros von Halikyai (Verr. 2,2,68-8I), in der der Propraetor C. Verres unter einem Vorwand einen Großteil seines consilium entließ, um den Angeklagten endlich schuldig sprechen zu können. Anders als Kirner möchte ich gerade hierin einen Beweis für eine Bindungswirkung des Gremiums sehen, wobei ich davon ausgehe, dass es sich, wenn nicht um gesetztes Recht, so doch zumindest um auctoritas bzw. um gebündelte auctoritates handelt, die so wohl kaum vom kognoszierenden Jurisdiktionsmagistraten ignoriert werden konnten. Auch die sorgfältig arrangierte Zusammensetzung des consilium im Kapitalprozess gegen Philodamos von Lampsakos (Verr. 2,I,63-85), die darauf abzielte, seine Verurteilung sicher zu stellen, lässt eher auf eine Verbindlichkeit des Votums schließen. Was allgemein das consilium betrifft, hat also Kunkels Satz weiterhin Gültigkeit: »Eine cognitio sine consilio galt als verwerflich, wenn nicht gar als rechtswidrig « (Die Magistratur, I995, I46). Der Prozess des Sthenius von Thermai (Verr. 2,2,83-I I 8) zeigt, wie sich Verres über die lex Rupilia, das Provinzstatut Siziliens seit I3 I v. Chr., und die vom Senat garantierte Autonomie der Stadt Thermai, die er selbst in seinem Edikt anerkannt hatte, hinwegsetzte. Rechtlich fixierte Satzungen wurden also von Verres mit Füßen getreten, auch eine Ediktsklausel, die Kirner anführt, gewährte ihm rechtlich diesen Spielraum nicht, da es sich bei dem Sthenius-Prozess nicht um die dort in Aussicht gestellte zweitinstanzliche Überprüfung von Urteilen handelte (Verr. 2,2,33: "wenn jemand eine Fehlentscheidung treffe, dann werde er den Fall untersuchen etc. «). Der Fall zeigt, 
dass sich Statthalter eigentlich an gesetztes Recht hielten. Aber das ist das sogenannte Recht des Stärkeren, der sich selbst durch das Recht bindet - oder es eben auch ignorieren kann. Wenn aber Statthalter allzu sehr über die Stränge schlugen, wie eben Verres, konnte dies in Rom von politischen Feinden etwa im Rahmen eines Repetundenverfahrens ausgenutzt werden. Letztlich spricht dieses Fallbeispiel eher für Mommsens Bild einer »selbstherrlichen Strafgerichtsbarkeit ", der ja mit Blick auf Verres explizit vom "schrankenlosen römischen Herrenrecht " spricht. Auch wenn ich Kirner in seiner plausiblen These über die informellen Handlungsspielräume statthalterlicher Politik zustimmen möchte, wird mir hier dem Recht doch zu wenig Gewicht beigemessen. Das Provinzstatut, bilaterale Verträge sowie das Statthalteredikt sind Teile einer Rechtsordnung, die Bestand hatte, auch wenn sie von einem Verres mit Füßen getreten wurde. Von einer »Rechtspraxis « kann im Sthenius-Fall nicht gesprochen werden, hier findet sich Willkür oder milder ausgedrückt, Herrschaft, die bestehendes Recht ignoriert.

Während für Mommsen die Statthalter der Republik »Verwalter des Herrenrechts « sind, sieht er in den kaiserzeitlichen Legaten » Träger der Reichsjustiz«. Die These will Kirner anhand der »Provinz« Judäa (6-66 n. Chr.) überprüfen. Zwar ist die Überlieferungslage zu Judäa gut, aber es liegt doch in der Verfasstheit administrativ-organisatorischer Strukturen und der Singularität religiöser Empfindsamkeiten eine Sonderform vor. Der rechtliche Status des Territoriums und die Stellung des Statthalters sind in der Forschung umstritten: Entweder wird Judäa als Provinz eines eigenständigen, nur dem Princeps verantwortlichen Statthalters angesehen oder bloß als Annex der Provinz Syrien, welches von einem dem syrischen Legaten untergeordneten Praefecten oder Procurator verwaltet wurde. Erkenntnisse zur 'Strafgewalt können also nicht unbedingt als herkömmliches Muster für römische Provinzialverwaltung auf andere Provinzen übertragen werden. Es überrascht in diesem $\mathrm{Zu}$ sammenhang, dass Kirner nicht die Münchner Dissertation von Peter Egger, CCrucifixus sub Pontio Pilator. Das >Crimen< Jesu von Nazareth im Spannungsfeld römischer und jüdischer Verwaltungs- und Rechtsstrukturen, aus dem Jahr I 997 zu kennen scheint.

Kirner geht davon aus, dass das Verhalten der Statthalter Judäas »allein von ihrer persönlichen Einschätzung der Lage, nicht aber von etwaigen rechtlichen Vorgaben abhing, nach denen sie sich zwangsläufig zu richten hätten " (189). Sicherlich hat das eigene Ermessen eine große Rolle gespielt, ja spielen müssen, wenn es um die Anwendung koerzitioneller Strafmaßnahmen ging; allerdings darf nicht außer Acht gelassen werden, dass wir es tatsächlich kaum mit unterschiedlichen Verhaltensweisen der jeweiligen Statthalter zu tun haben. Die einheitliche Vorgehensweise kann durchaus auf (gewohnheits-)rechtliche Grundlagen zurückgehen, hatte doch Rom schon seit mehr als drei Jahrhunderten Erfahrungen in der Wahrung der öffentlichen Ordnung. Gelungen sind in diesem Zusammenhang Kirners Ausführungen zum »informellen Kooperationsverhältnis « zwischen Statthalter und Führungsschicht der Provinzialbevölkerung. Hier hätte ich mir nur noch eine Berücksichtigung der Ausführungen von Egger (I00-I36) gewünscht.

Die unbestrittene Tatsache, dass der syrische Legat auch in Judäa seine Strafgewalt ausüben, ja sogar einen Praefecten vor das Kaisergericht bringen konnte, bedeutete, wie Kirner richtig ausführt, eine prekäre Situation für den Statt- 
halter, die so in anderen Provinzen nicht gegeben war. Bei all seinen Entscheidungen musste er negative Reaktionen des Legaten befürchten und damit auch mögliche Auswirkungen auf den kaiserlichen Hof, zudem war persönliche Einflussnahme durch prominente Provinziale auf den Kaiser selbst zu berücksichtigen. Abhängigkeiten, Eitelkeiten, Schwachheiten bestimmten politische und rechtliche Entscheidungen ohne dass wir aber rechtlich normierte Verwaltungsstrukturen auszuschließen bräuchten. Deutlich wird dies an den Prozessen gegen Jesus von Nazareth und Paulus aus Tarsus, denen Kirner jeweils ein eigenes Kapitel gewidmet hat. Richtig verweist er auf vielfältige herrschaftspragmatische Überlegungen, die einerseits zu einer Verurteilung des Galiläers oder andererseits zur Verschleppung des Verfahrens gegen Paulus geführt haben. Diese menschelnde Grauzone um das Recht herum hat es wohl zu allen Zeiten gegeben. Unabhängige, unbefangene »Träger der Reichsjustiz « werden sich vermutlich kaum finden lassen. Allerdings sieht auch Kirner in dem Vorgehen des Praefecten Pontius Pilatus zumindest im Strafverfahren vorgegebene, rechtlich fixierte Bahnen (290), während er dagegen eine rechtskräftige Wirkung der erfolgreichen Appellation im Fall des Paulus m. E. zu Unrecht bezweifelt (332).

Recht und Willkür: Wir wissen nicht, ob Johannes der Willkür eines selbstherrlichen Statt- halters zum Opfer gefallen ist oder aufgrund eines strafrechtlichen Verfahrens gekreuzigt wurde. Auch werden wir wohl nie erfahren, welche Interessen, Ängste oder Ressentiments zu diesem kapitalen Urteil beigetragen haben. So wie der Nagel im harten Holz des Ölbaumes mag sich auch der Statthalter bei seinen Entscheidungen gewunden und vielleicht auch gegen seine eigene Überzeugung verbogen haben, wie wir etwa am Beispiel der Prozesse gegen Jesus und Paulus sehen. Kirner hat mögliche anthropologische Konstanten in der politischen und sozialen Interaktion trefflich herausgearbeitet. Aber in dem Bestreben, die menschliche Seite des Regierens aufzuzeigen, schießt Kirner in seinem berechtigten Anliegen mitunter über das gewünschte Ziel hinaus. Das Recht verschwindet allzu sehr hinter dem damit notwendig zusammenhängenden menschlichen Ermessen. Rechtssätze, Rechtstraditionen werden in ihrer Bedeutung relativiert. Kirner läuft Gefahr, nun wiederum dem Recht kaum mehr ein Recht einzuräumen. Letztlich ist ihm zuzustimmen, wenn er feststellt: »Für eine Untersuchung der statthalterlichen Strafgewaltspraxis bedeutet die Trennung der rechtlichen von der politischen Ebene ebenso einen Anachronismus wie die Überbetonung der einen oder der anderen Ebene $«(344)$.

Loretana de Libero 